\title{
A Strategic Framework for Enterprise Information Integration of ERP and E-Commerce
}

\author{
Zaojie Kong, Dan Wang and Jianjun Zhang \\ School of Management, Hebei University of Technology, Tianjin 300130, P.R.China \\ kongzj@sina.com moyuhun_007@163.com zh_iianjunhaohao@163.com
}

\begin{abstract}
At the end of last century, E-commerce and ERP become the main methods to improve competitive power, but the integration of enterprise information of ERP and E-commerce system puzzled many enterprises, so in this article, the integration importance and the main problems that are faced in the integration process are analyzed firstly, and then the enterprise information integration frame of ERP and E-commerce based on Web Services and MultiAgent is proposed to solve platform heterogeneous, language differences, and achieve the two systems information alternation.
\end{abstract}

Keywords: Enterprise information integration, ERP, E-commerce, Agent, Web Services

\section{INTRODUCTION}

With the technology of computer and Internet swift and violent development, Ecommerce and ERP have become the main means of enterprise management. For the enterprise, E-commerce and ERP system like front and the rear relations, both are closely linked. For example, enterprises getting orders from e-commerce, should sent the order information immediately to the internal ERP system for Procurement, Production, Finance, Sales department arranging raw materials, capital, production and sale. If front-end E-commerce and the backstage ERP system come apart, this can cause lots of key information and data to be enclosed in mutually independent system. Sales order and market information obtaining from E-commerce platform cannot transmit into the backstage ERP system promptly; EC system can't read the product prices, customer information from ERP. The enterprise logistics, fund flow and information flow can't be unified organically, the uniformity, integrity and accuracy of data cannot be guaranteed. Departments cannot make the rapid, prompt, effective response to the customer, so it causes the enterprise working efficiency drop as well as the operation cost rise. Therefore, the E-commerce and ERP integration can not be ignored.

There are many differences in languages, platforms, communication protocols, and so on, between ERP and E-commerce. How to overcome the high cost of system integration arising from the differences is the key to solve this problem. For integration, information is the key. How to select the relevant information and how to achieve the two systems integrating fast and efficiently, which also don't allow to be ignored.

Please use the following format when citing this chapter:

Kong. 7.., Wang, D., 7hang, J., 2007, in IFIP International Federation for Information Processing, Volume 254. Research and Practical Issues of Enterprise Information Systems II Volume 1, eds. L. Xu. Tjoa A.. Chaudhry S. (Boston: Springer), pp. 701-705. 


\section{THE ENTERPRISE INFORMATION INTEGRATION FRAME OF ERP AND E-COMMERCE}

From above we know that achieving the systems integration is mainly to resolve heterogeneous and information alternation. Web Services technology based on XML is the best mean to resolve the heterogeneous, Agent can achieve information obtain, organization and transmission by its reactivity and intelligence.

Web Services [1] technology is the compilation of technologies (XML [2], Simple Object Access Protocol (SOAP) [3], Web Services Description Language (WSDL) [4], and Universal Discovery, Description, and Inventory (UDDI) [5]) that allow users to develop, catalog, and publish business services for delivery and use on the Web [6]. Web Services provide a distributed computing technology for revealing the business services of applications on the Internet or intranet using standard XML protocols and formats. The use of standard XML protocols makes Web Services platform, language, and vendor independent. Web Services eliminate the interoperability issues of existing solutions, such as CORBA and DCOM. Web Services are simpler, cheaper, based on open standards and more efficient, dynamic, and flexible [7].

The concept of Agent generates form Distributed Artificial Intelligent. It is a particular entity existing in the environment. Agent has following characteristics :(1) Autonomy: Agent has its own resources (data) and behavior control mechanism (process); it can operate without people. (2) Sociability: Agent and others (including people) can communicate with some language for coordination and cooperation. (3) Reactivity: Agent observes the environment, make responses to the change. (4) Proactivity: Agent not only reacts to the environment, but also accepts certain message, to take the initiative action, which is goal-oriented behavior. (5) Intelligence: Agent has intelligence including reasoning and learning by itself.

It is obvious that the integration problem can be solved by using web services and agent, the enterprise information integration frame is as shown in figure 1.

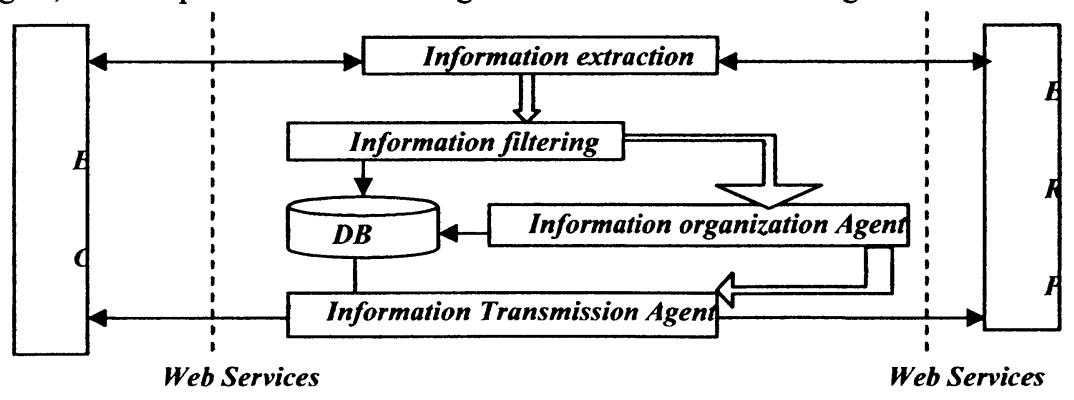

Figure 1. The Enterprise Information Integration Frame of ERP and Ecommerce

The frame consists of Information extraction Agent, Information filtering Agent, Information organization Agent and Information Transmission Agent. Web services shields platform heterogonous, data and language differences, the information extraction agent communicate with EC and ERP systems, and extract the integrating information, but there also may be some dirty data ,so the data should be filtered into 
DB by Information filtering Agent, then all information is organized by Information organization Agent, at last, the integrating information can be transferred quickly and efficiently by Information transmission Agent, therefore the integration of ERP and E-commerce can be achieved.

\section{SYSTEM WORK MECHANISM}

\subsection{Web Services}

All kinds formats information from the two systems can be transformed into XML by Web Services, so all information can be transferred. And all the output information can be transformed the right format by XSLT. Web Services provide the sharing platform for the information systems, all information can be read.

\subsection{Agents Function}

\subsubsection{Information Extraction Agent}

Information extraction Agent mainly learns the extraction rules and extracts the integrating information. Agents who want to achieve its functions depend on Knowledge Base and database. The knowledge base of Information extraction Agent includes the domain knowledge base, the extraction request base and the extraction rule base. The domain knowledge base is the knowledge of the basic concept, attribute, entity, rules and so on, which is contained in the field of the extraction information. The extraction request base records use history, memories the extraction requests. When meet the similar request, it will take the optimized request from the base to users. The extraction rule base memory extraction rules. Its main body can be divided into two parts, one is the information format knowledge is relevant to description format; the other is semantic knowledge to describe the information content. When there is new information, Information extraction Agent starts the system by autonomy, accepts the initial extraction request, optimizes the extraction request constantly, learns the rules, then extracts the information. At last results will be taken to deal with by information filtering agent.

\subsubsection{Information Filtering Agent}

The goal of Information filtering Agent is to help users to eliminate the information that they don't want to get. Information filtering Agent filters the extraction information, and then put the information into database. It can automatically obtain external dynamic information; then information is filtered into Eigen value and 
special symbols. These values or symbols are stored into database; they can directly lead to action or are processed in deeper level by information organization agent after further polymerization.

For lots of relevant extraction information, Information filtering Agent must filter the data according to the system needs. At present, there are four information filter methods which usually are used [8]: based on keywords vector information filtering methods, based on articles information filtering methods, Multi-Agent filtering methods and evolutionary information filtering method. System can take based on keywords vector information filtering methods. The system needs and the extraction information can be described in VSM, by calculating the two models similarity, when the similarity is over a threshold, put the information into database, otherwise give it up.

\subsubsection{Information Organization Agent}

Information organization Agent organizes the information in database. It will change information format and manage the information resource by organization, addition, deletion, modify, sorting and inquiry, etc. It not only makes a response quickly, but also manages effectively.

It organizes the information according to the thought of Data warehouse. Data warehouse is the data aggregation which is facing theme, compositing, non-volatile, and supporting the decision-making of enterprises with the time. The data in Data warehouse is from many different data resources. When the data gets into Data warehouse, it will be converted, reformatted, rearranged and exchanged. The result is that the data in Data warehouse has single physical corporate image, so we can organize the data according the characteristic of Data warehouse.

The focus of Data warehouse design is theme, which is always decided by the enterprise needs. When theme selected, it is decided database dimension, dimension attributes and facts table attributes based on granularity. So the filtered information can be organized effectively and transferred into EC and ERP systems efficiently and accurately.

\subsubsection{Information Transmission Agent}

Information Transmission Agent mainly completes initiative transferring. It searches the integration information, and provides the new inquiry result into EC and ERP systems according to information integration needs. It will notify the systems timely when monitoring the dynamic changes of data, as information expired, update or lost, to achieve the enterprise information alternating.

\subsection{Agent Communication Pattern}

The system uses SOAP communication mechanism to achieve interoperability between agents. SOAP is a cross-platform protocol standard, it can allocate the object and communicate at heterogeneous platforms by combination with XML. 
Figure 2 is a simple agent communication model. Information extraction agent is the sender of SOAP message, Information Transmission agent is the receiver. Information filtering Agent and Information organization Agent is also the sender and the receiver. The sender sends the SOAP message to the receiver; the receiver receives the message, parses the message and obtains the content.

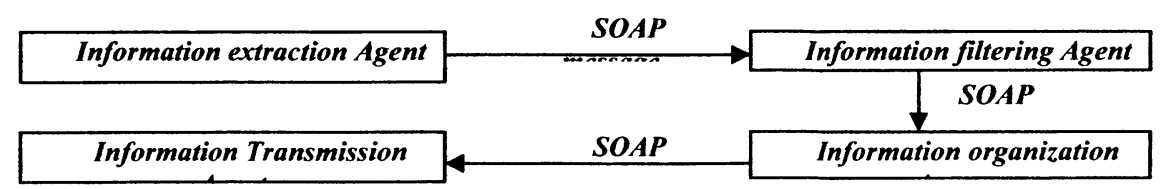

Figure 2. Agent Communication Model Based on SAOP

\section{SUMMARY}

The enterprise information integration system takes full advantage of Web Services to solve platform heterogeneous and the intelligence of agent. It greatly improves the information transmission speed and accuracy. It only puts forward a conceptual model. The specific construction strategies and methods need summarizing and exploring in practice. However, analysis indicates that the integration strategy is a new idea for resolving the problem of enterprises information isolated islands, it also can be used in other enterprise information systems integrating.

\section{REFERENCES}

1. R. Bhatti, E. Bertino, and A. Ghafoor, XML-based specification for web services document security, IEEE Comps. Volume 37, Number 4, pp.41-49, (2004).

2. T. Bray, J. Paoli, and C.M. Sperberg-McQueen, $X M L$ extensible markup language $(X M L)$ 1.0. (2004). http://www.w3.org/TR/2004/REC-xml-20040204 (Accessed March 4, 2007).

3. Anonymous, W3C.SOAP1.2,W3C(2003). http://www.w3.org/2000/XP/Group (Accessed February 4, 2007).

4. Anonymous, W3C.WSDL2.0,W3C(2004). http://www.w3.org/2002/ws/desc (Accessed March 4, 2007).

5. Anonymous, UDDI ORG.UDDI3.0 W3C (2003). http://uddi.org/pubs/uddi v3.htm. (Accessed March 4, 2007).

6. K.K.E Venugopal and J.G. Kupper, Web services and EAI-what 'it' is (2002). http://www.webservices.org/article-php?sid=421 (Accessed March 4, 2007).

7. G. Samtani and D. Sadhwani, EAI and web services easier enterprise application integration? (2002). http://www.webservicesarchitect.com/content/articles/samtani01.asp (Accessed February 4, 2007).

8. H. Chen, $\mathrm{W}$. $\mathrm{Li}$, and $\mathrm{S}$. Liu, the Design and Implementation technology of intelligent information filtering Agent, Guangdong University of Technology Journal. Number 9 , pp.33-35, (2001). 